\title{
Momento de riego y tamaño de bulbo en el rebrote y floración del Amancay (Ismene amancaes. Ruiz \& Pavón)
}

\author{
Irrigation time and bulb size in the resprouting and flowering of Amancay \\ (Ismene amancaes. Ruiz \& Pavon)
}

${ }^{1}$ José Alfredo Palacios Vallejo

\begin{abstract}
Resumen
El objetivo del presente experimento fue determinar la influencia del tiempo de reposo antes del riego sobre el rebrote y floración en tres diferentes tamaños de bulbo de Ismene amancaes. Los bulbos cosechados, después de la senescencia de sus hojas, fueron seleccionados por tamaño (grandes: $80.2 \pm 19.3 \mathrm{~g}$, medianos: $58.08 \pm 14 \mathrm{~g}$ y pequeños: $18.78 \pm 6.5 \mathrm{~g}$ ) y colocados en envases conteniendo turba seca para permanecer en reposo durante 3, 2 y 1 meses, antes de ser regados. Los resultados muestran que cuanto mayor fue el retraso del riego, menor fue el tiempo para el rebrote en los tres tamaños de bulbo. Sin embargo, solo hubo diferencias significativas en los bulbos grandes y medianos regados después de dos y tres meses, frente a los regados después de un mes. En relación a la floración, los bulbos de mayor tamaño fueron los que tuvieron el mayor porcentaje de floración, pero solo mostraron diferencias significativas con los bulbos pequeños. El momento de riego también tuvo influencia sobre el número de bulbos con flor. Los bulbos regados luego de tres meses de reposo, fueron los que más florearon con diferencias significativas con los de un mes de reposo, pero sin diferencias con los de dos meses. Los tres tamaños de bulbo, sin considerar los momentos de riego, rebrotaron entre los 269 - 318 días. Esto significa que todos los bulbos, deben completar primero su desarrollo interno (diferenciación foliar e iniciación floral) antes de estar listos para rebrotar bajo condiciones ambientales externas favorables.
\end{abstract}

Palabras clave: Ismene amancaes, riego, tamaño de bulbo, rebrote, floración.

\begin{abstract}
The objective of this experiment was to determine the influence of the length of the rest period before irrigation on the resprounting in three different bulb sizes of Ismene amancaes. Bulbs were harvest after senescence of their leaves, classified by size (large: $80.2 \pm 19.3 \mathrm{~g}$, medium: $58.08 \pm 14 \mathrm{~g}$ and small: $8.78 \pm 6.5 \mathrm{~g}$ ), and placed in containers with dry peat moss for resting for 3, 2 and 1 months before being irrigated. The results show that when the time before resuming irrigation was longer, the time to resprout was shorter for the three bulb sizes. However, only in large and medium bulbs, irrigation after two and three months had significant differences with bulbs irrigated after one month. In relation to flowering, the larger bulbs had the highest percentage of flowering, but with significant differences only with small bulbs. The time of irrigation also had influence on the number of bulbs in bloom. Irrigating bulbs after three months of rest resulted in more flowers with significant differences with one month of rest, but no differences with two months. The three bulb sizes, without considering irrigation times, resprouted between $269-318$ days. This means that large, medium and small bulbs should first complete their internal development (leaf differentiation and floral initiation) before they are ready for resprouting under favorable external environmental conditions.
\end{abstract}

Key words: Ismene amancaes, irrigation, bulb size, resprout, flowering.

\section{Introducción}

En el Perú, existen 136 especies de la familia Amarilidaceae adaptadas a ambientes xéricos, de las cuales 54 son endémicas. Una de ellas es el 'amancay' (Ismene amancaes), que se desarrolla en lomas de la costa central del país y es considerada un símbolo de la ciudad de Lima.

Sin embargo, poco se ha hecho por determinar cuáles son los factores que influyen sobre el crecimiento vegetativo y diferenciación floral en bulbos de amancay.
Aún falta información sobre cuál o cuáles son los factores ambientales (temperatura, humedad, intensidad luminosa, longitud del día, etc.) que tienen finalmente un efecto directo sobre su fenología. Según Halevy (1990) cuando los bulbos son producidos bajo condiciones diferentes a su hábitat natural, su crecimiento puede verse alterado. Por lo tanto, es importante evaluar los efectos de la temperatura, luz, agua, nutrición, reguladores de crecimiento y otros factores ambientales que intervienen en su crecimiento. Con esta información será posible ampliar significativamente el manejo de esta especie en 
diferentes épocas del año.

Dentro de los factores ambientales, la temperatura ha sido considerada como el factor más importante en el crecimiento, desarrollo e iniciación floral de muchas especies bulbosas. Sin embargo, para el caso específico de Ismene amancaes, el factor humedad parece tener una influencia mucho mayor, especialmente al momento del rebrote y floración de esta especie.

El amancay a diferencia de otras especies bulbosas, originarias de otras latitudes, comienza a rebrotar con la presencia de la humedad atmosférica procedente de las neblinas que ocurren durante los meses de invierno. Brack (1999), describe al amancay (Ismene amancaes) como una planta herbácea, bulbosa, que solo se activa en la estación fría y nublada (mayo a setiembre) de invierno.

Cuando estas condiciones desaparecen, los bulbos entran en un periodo de dormancia hasta nuevamente encontrar condiciones favorables.

Fenología del amancay. Hartmann, Kester, Davies, and R. Geneve.(2002) afirman que los bulbos en general tienen un ciclo de desarrollo característico, que comienza con su iniciación como meristemo y termina en la floración y producción de semillas. Este ciclo de desarrollo general, está compuesto de dos estados: (a) vegetativo y (b) reproductivo. En el estado vegetativo, el bulbo crece hasta alcanzar un tamaño y peso máximo, lo que le permite llegar a floración. En el estado reproductivo, se incluye la inducción e iniciación de flor, con la diferenciación de las partes de la flor, la elongación del brote floral y finalmente la antesis (floración). Brito (2006), sostiene que entre los factores más importantes, que afectan el crecimiento y desarrollo de un bulbo, están: la temperatura, la luz y la humedad. Estos tres factores son los utilizados en el forzamiento de bulbos, debido a que actúan directamente sobre el enraizamiento, desarrollo floral, elongación de brotes y de bulbos. Considera que la temperatura es el factor externo más importante, pues controla el crecimiento, el desarrollo, la floración, la dormancia y la madurez fisiológica de los bulbos. La intensidad de luz y el fotoperiodo también pueden afectar ciertos procesos fisiológicos, como la fotosíntesis, el aborto y abscisión de flores.

Para una mejor comprensión de los factores ambientales que afectan la fenología del amancay, se han considerado en la presente investigación cinco etapas: una primera etapa donde se inicia la fase vegetativa (rebrote y desarrollo foliar), una segunda etapa en que se da la fase reproductiva (emergencia del escapo floral, floración, y fructificación), una tercera etapa donde se acumulan fotosintatos en el bulbo, una cuarta donde la planta empieza su senescencia (muerte de flores y hojas) y finalmente una quinta etapa donde el bulbo entra en "reposo" o "dormancia".

Primera Etapa. Es el inicio de la fase vegetativa, donde emergen las primeras hojas. En esta fase, la temperatura siempre ha sido considerada como un factor muy influyente en el rebrote de muchas especies bulbosas. Borys, Leszcynska-Borys, and Galvan. (2008) en su estudio de especies mexicanas de Hymenocallis pertenecientes a grupos xerofíticos y meso-hidrofíticos, sugieren que el inicio de la emergencia de hojas y la finalización de los escapos está relacionada a la variación de la temperatura del suelo. Borys et al. (2009) analizaron las características particulares del grupo xerofítico de Hymenocallis y establecieron que durante la época en que la temperatura del suelo era más baja (septiembre febrero) los bulbos entraban en dormancia, mientras que en las épocas en que la temperatura era más alta (marzo - junio) los bulbos rebrotaban, floreaban y formaban semilla. Sin embargo, para el caso de Ismene amancaes, la humedad parece ser un factor determinante para el rebrote de los bulbos, al margen de la temperatura. Torres y López (1981), señalan que la presencia de neblinas y garuas, incrementa la humedad del aire y del suelo. La disponibilidad de agua en ese periodo, fue suficiente para que se inicie una vegetación temporal. Además de los valores máximos de humedad, también se tiene valores mínimos de temperatura del aire y del suelo. La interacción de estas dos variables, crea condiciones óptimas para la germinación y rebrotamiento de especies herbáceas y supraterrestres. La productividad, en consecuencia, alcanza sus valores máximos durante este periodo, constituyéndose en la época de auge florístico y vegetacional de las Lomas. Ferreyra (1979), reafirma que el desarrollo de la vegetación en lomas está esencialmente ligado a las lluvias y garuas de invierno (junio a agosto). Agüero (2002), refiere que el rebrote de los amancays se inicia en el primer mes de almacenamiento de humedad edáfica y en relación directa con ella. Agüero y Suni (1999), mencionan que la humedad relativa y el contenido de humedad del suelo son los que determinan el inicio del rebrote del amancay.

Segunda Etapa. Es el inicio de la fase reproductiva, donde aparece el escapo floral, las flores y los frutos. Es una etapa donde también se incrementa el área foliar, lo que favorece la fotosíntesis y la fabricación de reservas (fotosintatos) para el desarrollo de la flor. Wiebe (1994), afirma que en la mayoría de geófitas, el factor más importante para controlar floración es la termoperiodicidad estacional. Según Wang y Roberts (1983), la temperatura desempeña un papel básico en la progresión del crecimiento vegetativo a la formación de la yema floral. Para las diversas fases de desarrollo se estableció una temperatura óptima, determinada por el tiempo más corto en que llegaba a florear un bulbo. En general, una desviación de las temperaturas optimas o una prolongación de cualquiera de las temperaturas, produjo un aumento en el tiempo requerido para florecer y en algunos casos impidió completamente el desarrollo de la flor. De Hertogh (1996), observó que la velocidad de floración en Leucojum aestivum (Amaryllidaceae), era controlada por las temperaturas; por lo tanto, estos bulbos podían ser forzados para acelerar o retrasar el inicio de su floración. Mori, Imanishi y Sakanishi (1991), investigaron el efecto de la temperatura en la floración de 
Hymenocallis speciosa y encontraron que la formación de flores se inhibió en plantas mantenidas a $25^{\circ} \mathrm{C}$, pero fue promovida en plantas transferidas a temperaturas de $15^{\circ} \mathrm{C}$ o $20^{\circ} \mathrm{C}$, la temperatura de almacenamiento también tuvo un efecto sobre el tamaño de flor y escapo. Para el caso de Ismene amancaes, Suni, Pascual y Jara. (2011), refieren que la diferenciación de las yemas florales se inicia luego de que se han formado las hojas que saldrán el siguiente año y en un periodo de máximo descenso de la humedad edáfica (noviembre). En los meses de agosto y setiembre se obtuvieron los mayores valores de humedad del suelo para declinar precipitadamente en noviembre, periodo en el que se considera se inicia la diferenciación de las yemas florales.

Tercera Etapa. Es la etapa donde el follaje continúa activo a pesar de la senescencia de la floración. Gracias a ello las hojas, a través de la fotosíntesis, ayudan a acumular reservas en el bulbo. Esta acumulación de fotosintatos dependerá de las condiciones de humedad y temperatura presentes en el lugar. Si las condiciones de humedad disminuyen y las temperaturas aumentan, el follaje comenzará su senescencia reduciendo la acumulación de fotosintatos, lo que causará un menor crecimiento del bulbo. Si las condiciones de humedad permanecen más tiempo y las temperaturas se mantienen, el proceso de acumulación de reservas será mayor y el tamaño del bulbo aumentará. Rossa y Von Willert (1999) mencionan que la translocación de reservas y nutrientes desde las hojas hacia el bulbo son un componente importante en la estrategia de vida de las geofitas, pues asegura la utilización eficiente de los minerales, permitiendo que puedan desarrollarse en suelos pobres de nutrientes. Dafni, Cohen, and Noy-Meir (1981), indican que las reservas necesarias para la floración se acumulan del año anterior; si las reservas son insuficientes, la floración no ocurre el siguiente año y todas las reservas son utilizadas para los órganos fotosintéticos. Morales, Schiappacasse, Peñalillo y Yañez (2009), refieren que al finalizar su ciclo de crecimiento, todos los tamaños de bulbo experimentan una variación con respecto a su peso inicial; los pesos en bulbos pequeños aumentan, pues no alcanzan el tamaño mínimo para florear; por el contrario, los bulbos de mayor tamaño que florean y producen flores grandes, sufren una reducción en su peso. Rees (1972) menciona que las altas temperaturas después de la floración pueden tener importantes efectos sobre la aceleración del proceso de senescencia, especialmente en países áridos, donde suele ocurrir con gran rapidez. En el caso de amancay, Suni et al. (2011) mencionan que el periodo de acumulación de fotoasimilados, que incrementa la biomasa al bulbo, dura solamente dos meses (agosto y setiembre). Sin embargo este periodo es determinante para el éxito reproductivo del siguiente periodo.

Cuarta Etapa. Esta etapa se inicia con la senescencia de hojas y finaliza al entrar los bulbos en "reposo" o "dormancia", influenciados por la temperatura y ausencia de humedad. En esta etapa cesa todo crecimiento externo del bulbo. Hartmann et al. (2002), señalan que el fin de la fase vegetativa se reconoce por el secamiento del follaje y la maduración del bulbo; después de ese periodo, los bulbos ya no aumentan ni en peso ni en tamaño. Las raíces se desintegran y el bulbo entra en un periodo aparente de "reposo". Dafni et al. (1981), piensan que factores como las temperaturas del aire y del suelo, la disminución de la humedad del suelo y el fotoperiodo, juegan un rol preponderante en la senescencia e inicio de la dormancia tanto de verano como de invierno. En el caso de amancay, Agüero y Suni (1999), observaron una marcada senescencia, cuando la humedad relativa y el contenido de humedad del suelo decrecieron.

Quinta Etapa. Se inicia con el "reposo" o "dormancia", donde se suspende temporalmente todo crecimiento visible. De Hertogh y Le Nard (1993) lo definen como un complejo y dinámico proceso fisiológico, morfológico y bioquímico, en el que no hay aparentemente cambios externos o de crecimiento; sin embargo, internamente se pueden presentar muchos eventos fisiológicos y/o morfológicos. Kemenetsky (1994) propuso el término "desarrollo interno del bulbo" para describir mejor la "dormancia de verano" en especies bulbosas; durante este periodo, la actividad fisiológica tiene lugar debajo del suelo, donde frecuentemente se incluye la diferenciación celular y la iniciación floral. Rees (1972), indica que cuando la parte aérea ha muerto y no hay partes activas por encima del nivel del suelo, la planta aparentemente esta dormante. Sin embargo, debajo del suelo, existe actividad debido a que los ápices pueden estar iniciando sus primordios florales; esta dormancia, si es posible el término, es una forma de "dormancia de verano" debido a que es una respuesta a las severas condiciones de calor y sequia de su hábitat natural; el crecimiento normal solo se reasume cuando la planta ha pasado a través de las bajas temperaturas de invierno. El mismo Rees (1992), señala que los factores que promueven la "dormancia" de los bulbos son diversos y difieren de acuerdo al hábitat donde se desarrollan las especies. Es entonces el hábitat, quien determina las necesidades fisiológicas de las especies; por ejemplo, las especies bulbosas de zonas templadas (Allium e Iris) necesitan usualmente bajas temperaturas $\left(9\right.$ a $13^{\circ} \mathrm{C}$ ) para una diferenciación floral óptima; las especies con ciclos termoperiódicos, originarias de regiones irano - turánica (Tulipa y Hyacinthus), necesitan de temperaturas relativamente altas para su diferenciación $\left(17\right.$ a $\left.25^{\circ} \mathrm{C}\right)$ y un periodo de bajas temperaturas $\left(4\right.$ a $\left.9^{\circ} \mathrm{C}\right)$ para la elongación del tallo floral y la antesis; mientras que las geofitas de regiones mediterráneas que florean en otoño (Crocus), están adaptadas a condiciones de ambientes cálidos. Kamenetsky y Okubo (2013), mencionan que en el caso de regiones semiáridas como el Cabo (Sudáfrica), donde las mayores precipitaciones se dan en los meses de invierno, la mayoría de geofitas son estacionales y permanecen dormantes durante la estación seca. La ventaja de estas geofitas, es su habilidad para sobrevivir en los cortos periodos de sequía y responder rápidamente cuando las condiciones de humedad mejoran. Si los periodos de sequía se prolongan, se puede 
limitar el reabastecimiento del bulbo y comprometer su floración. Borys et al. (2009) afirman que plantas de Hymenocallis bajo riego, presentan diferentes periodos de dormancia, juzgado a través de la ausencia de hojas y escapos. Los bulbos de estas plantas pueden tener una extendida dormancia profunda, o pueden tener sus raíces o bulbos necesidades térmicas variables para comenzar a producir hojas y escapos. En el caso de Ismene amancaes, los bulbos permanecen dormantes en épocas de sequía y rebrotan solo en presencia de la garua invernal. Según Suni et al. (2011) cuando declina el contenido de humedad del suelo y se incrementa la temperatura, ocurre el inicio de las yemas florales.

\section{La humedad en el rebrote del amancay}

Hartmann et al. (2002) refieren que hay cierto número de bulbos procedentes de regiones tropicales, cuyo ciclo de desarrollo está condicionado por un ciclo climatológico húmedo - seco, en lugar de estarlo por un ciclo de temperaturas frio - cálido.

Para determinar los tiempos de rebrote, desarrollo y floración del amancay, se han llevado a cabo múltiples estudios a lo largo de muchos años. En ellos, se han señalado diferentes épocas de floración, pero siempre asociados a la presencia de una alta humedad relativa. Weberbauer (1945), indica que la floración en Hymenocallis amancaes (actualmente Ismene amancaes) se inicia en el mes de junio cuando reverdecen las lomas y se ven campos con flores amarillas desde Asia hasta Huacho y en el mes de agosto generalmente ya no tienen flores. Aguilar (1974), refiere igualmente que en las lomas de Atocongo y Lurín, el amancaes se encuentra en plena floración en el mes de junio y en fructificación el mes de julio. Torres y López (1981) mencionan que la etapa vegetativa del amancay se inicia en mayo, seguida de una etapa reproductiva en junio, continuando su desarrollo vegetativo hasta septiembre. Esto coincide con la mayor incidencia de neblinas advectivas y garuas de julio a setiembre, que determinan máximos valores de humedad en el aire $(85-100 \%)$ y en el suelo $(12-15 \%)$, pero contrariamente, se dan los valores mínimos de temperatura del aire $\left(13^{\circ}-16^{\circ} \mathrm{C}\right)$ y del suelo $\left(15^{\circ}-18^{\circ} \mathrm{C}\right)$. Agüero y Suni (1999), confirman que el amancay, bajo condiciones normales de humedad, rebrota en junio, florece a fines del mismo mes manteniendo sus flores hasta julio, a fines de agosto fructifica y en septiembre comienza a producir semillas. Sin embargo, bajo condiciones del Fenómeno del Niño 1997 - 1998, los bulbos rebrotaron a inicios de agosto; esto debido a que la humedad del suelo alcanzó el $8.3 \%$, con temperaturas máximas de $20.5^{\circ} \mathrm{C}$ y mínimas de $16.4^{\circ} \mathrm{C}$, con una humedad relativa de 98,90 y $95 \%$ a las 7 , 13 y 19 horas respectivamente. La senescencia se alcanzó a fines de setiembre, cuando la humedad del suelo bajó a $7.2 \%$ y en octubre a 3.3\%. Cano et al. (1999) mencionan que en condiciones normales o años sin "El Niño", la vegetación de lomas de la costa central alcanza su óptimo en los meses de agosto y setiembre como consecuencia de la humedad generada por la condensación de las neblinas invernales. Por otro lado, Ochoa (2008), señala que el amancay en Santiago de Tuna, provincia de Huarochiri, departamento de Lima, modifica su época de florecimiento del solsticio del 24 de junio al solsticio del 24 de diciembre, y considera que esto se debe al cambio de piso ecológico, a la mayor altura (2000 msnm) y a las densas neblinas previas a las lluvias de temporada.

\section{El Tamaño de bulbo en el rebrote del amancay}

Halevy (1990) refiere que adicionalmente a los factores ambientales, el tamaño del bulbo generalmente determina si se van a producir flores o no. Incluso bajo condiciones inductivas, los bulbos por debajo del tamaño crítico normalmente no florean. Por encima del tamaño crítico, la producción de flores (número de brotes florales) y su calidad (longitud del tallo, numero de flores por inflorescencia) se ve frecuentemente incrementada por el tamaño de bulbo. Le Nard y De Hertogh (2002), indican que el tamaño de bulbo (cantidad de reservas), constituye uno de los primeros criterios de calidad. Es conocido que la mayoría de los bulbos de flor alcanzan un tamaño crítico antes que ocurra la inducción floral. Generalmente los bulbos grandes producen plantas vigorosas con muchas flores y/o de gran tamaño. Theron y De Hertogh (2001) mencionan que en varias especies, la habilidad de florear está directamente relacionada al tamaño del órgano geofitico, el cual varía de especie a especie. Por ejemplo, dentro de la familia Amarilidaceae el Hippeastrum alcanza su floración cuando sus bulbos tienen una circunferencia aproximada a los $20 \mathrm{~cm}$ (6 $\mathrm{cm}$ de diámetro). En Scadoxus se tiene floración cuando la circunferencia del órgano geofitico es de $15 \mathrm{~cm}(4.5$ $\mathrm{cm}$ de diámetro), mientras que en bulbos de Eucharis se debe tener una circunferencia de $3-5 \mathrm{~cm}(1 \mathrm{~cm}$ de diámetro) antes de que ocurra la floración. Hanks y Jones (1987), seleccionaron bulbos de Hymenocallis x festalis de diferentes tamaños: desde $9-10 \mathrm{~cm}$ de circunferencia hasta más de $18-19 \mathrm{~cm}$. Allí observaron que solo el 25\% de los bulbos de menor tamaño $(9-10 \mathrm{~cm})$ produjeron flores, mientras que por lo menos el $90 \%$ lo hizo en bulbos con una circunferencia mayor a los $13 \mathrm{~cm}$. El periodo de floración fue ligeramente más largo en bulbos de mayor tamaño (43 días en bulbos pequeños, 49 días en bulbos grandes).

En el caso del amancay, Carillo (2004), indica que se puede diferenciar dos etapas fenológicas: los bulbos juveniles en las que rebrotan solo las hojas y el desarrollo es solo foliar hasta su senescencia; y los bulbos adultos, en los que se desarrollan las hojas y luego sucede la floración o primero emergen los botones florales y estos se desarrollan al mismo tiempo que las primeras hojas. La fenología en el bulbo adulto es tan rápida que la planta en un mes puede haber logrado el desarrollo foliar, floración y fructificación.

El presente trabajo busca determinar si el factor humedad a través de diferentes momentos de riego, puede promover, en diferentes épocas del año, el rebrote, desarrollo y floración en bulbos de amancay de diferente tamaño. 


\section{Materiales y Métodos}

El presente experimento se realizó en el Programa de Investigación en Ornamentales de la Universidad Nacional Agraria La Molina, ubicado en la provincia de Lima, departamento de Lima, Perú. La fecha de inicio del experimento fue el 18 de julio del 2014 y finalizó el 15 de junio del 2015. En el mes de julio se cosecharon bulbos de Amancay (Ismene amancaes) luego de la senescencia de sus hojas y se les seleccionó por su tamaño en: 60 bulbos grandes $(16-18 \mathrm{~cm}$ de circunferencia y peso promedio de $80.3 \pm 19.3 \mathrm{~g}), 60$ bulbos medianos $(9-15.9 \mathrm{~cm}$ de circunferencia y peso promedio de $58.08 \pm 14 \mathrm{~g}$ ) y 60 bulbos pequeños $(3.0-8.9 \mathrm{~cm}$ de circunferencia y peso promedio de $18.78 \pm 6.5 \mathrm{~g})$. En total se seleccionó 180 bulbos. Los bulbos cosechados (60 grandes, 60 medianos y 60 pequeños), fueron plantados en envases conteniendo turba, donde permanecieron sin riego hasta el inicio del experimento. Pasados 30 días (17/8), se seleccionó un primer grupo de bulbos: 20 grandes, 20 medianos y 20 pequeños, que comenzaron a ser regados semanalmente. A los 60 días (16/9), se seleccionó un segundo grupo de bulbos: 20 grandes, 20 medianos y 20 pequeños, para recibir también riegos semanales. Finalmente, el grupo restante de bulbos (20 grandes, 20 medianos y 20 pequeños), comenzaron a ser regados a los 90 días de cosechados (15/10). Todos los bulbos fueron plantados en un sustrato de $100 \%$ turba desinfectada (Distichia muscoides). Todos los tratamientos fueron colocados bajo sombra (50\%) y a temperatura ambiente (Tabla 1$)$. a la prueba de significación de Duncan con un nivel de significancia de 0.01 .

\section{Resultados y discusión}

Evaluación del tiempo de rebrote según el tamaño de bulbo de "Amancay"

Se comparó independientemente los tres diferentes tamaños de bulbo seleccionados (grandes, medianos y pequeños), sin considerar los momentos de riego, para observar el tiempo de rebrote en cada uno de ellos.

Tabla 3. Tiempo de rebrote de tres tamaños de bulbo de Amancay

\begin{tabular}{cc}
\multicolumn{2}{c}{ Amancay } \\
\hline Tamaño de bulbo & Promedio de días para el rebrote \\
\hline Grandes & $290.71 \mathrm{a}$ \\
Medianos & $283.42 \mathrm{a}$ \\
Pequeños & $284.78 \mathrm{a}$ \\
Promedio & $286.30 \mathrm{a}$ \\
\hline
\end{tabular}

En la Tabla 3, se observa que no hubo diferencias significativas entre los diferentes tamaños de bulbo. El número de días para el rebrote fue en promedio de 286.30 días (9.5 meses). Estos resultados muestran que los bulbos grandes, medianos y pequeños deben tener un periodo

Tabla 1. Promedio de temperaturas máximas y mínimas durante investigación

\begin{tabular}{lcccccccccc}
\hline \multicolumn{1}{c}{ Temperatura } & Jul & Ago & Set & Oct & Nov & Dic & Ene & Feb & Mar & Abr \\
\hline Máxima & 19.5 & 19.4 & 19.5 & 21.3 & 23.0 & 23.7 & 26.0 & 29.0 & 28.2 & 27.5 \\
Mínima & 15.6 & 14.0 & 14.2 & 14.7 & 15.8 & 17.7 & 19.7 & 20.4 & 19.8 & 17.7 \\
\hline
\end{tabular}

Tabla 2. Tratamientos

Inicio del riego Numero de bulbos por tamaño
$\mathrm{T}_{1}: 3$ meses de 20 Grandes 20 Medianos 20 Pequeños
plantación

$\mathrm{T}_{2}: 2$ meses de 20 Grandes 20 Medianos 20 Pequeños
plantación

$\mathrm{T}_{3}: 1$ mes de 20 Grandes 20 Medianos 20 Pequeños
plantación

Se empleó un diseño estadístico completamente al azar, con 20 repeticiones (bulbos) por tratamiento, donde se evaluaron independientemente cada uno de los tamaños de bulbo. A los resultados del experimento se les realizó un Análisis de Variancia y las medias fueron sometidas aproximado de 286.3 días para completar su desarrollo interno (diferenciación foliar e iniciación floral) y estar listos para rebrotar al momento en que las condiciones ambientales externas sean favorables. Esto se aproxima a lo encontrado en investigaciones realizadas por Suni et al. (2011), donde se indica que el periodo de diferenciación y desarrollo de las estructuras reproductivas, abarca nueve meses (noviembre a julio).

Sin embargo, en el presente experimento hay que tomar en cuenta que el tiempo de rebrote, en los tres tamaños de bulbo, se inició entre los 269 - 318 días ( 9 - 10. 5 meses), lo que permite suponer que algún otro factor (temperatura o humedad) pudo tener influencia sobre este desarrollo.

Evaluación del tiempo de rebrote de bulbos de "amancay" según el momento de riego

Se evaluó independientemente los tres momentos de riego, sin considerar el tamaño de bulbo, para determinar 
su influencia sobre el tiempo de rebrote.

Tabla 4. Tiempo de rebrote de bulbos de Amancay en tres momentos de riego

\begin{tabular}{cc}
\hline Momento de riego & $\begin{array}{c}\text { Promedio de días pare el } \\
\text { rebrote }\end{array}$ \\
\hline 3 meses & $278.21 \mathrm{a}$ \\
2 meses & $285.90 \mathrm{a}$ \\
1 mes & $297.76 \mathrm{~b}$ \\
\hline
\end{tabular}

Los resultados muestran que conforme el momento de riego se fue retrasando $(1,2$ y 3 meses después de la plantación), el rebrote se dio en un menor número de días (Tabla 4). Sin embargo, estadísticamente solo los bulbos regados 3 y 2 meses después de ser plantados, tuvieron diferencias significativas (278.21 y 285.9 días) con los bulbos regados al mes de sembrados (297.76 días). Como se indicó anteriormente, el periodo necesario para la diferenciación y desarrollo de estructuras vegetativas y reproductivas es de aproximadamente nueve meses (270 días). Los resultados de la presente investigación, muestran que el rebrote estuvo siempre por encima de este periodo y que los tratamientos de riego solo lograron igualarlo o retrasarlo ligeramente.

Cuando Ferreyra (1993), Agüero y Suni (1999) y Agüero (2002), indican que el rebrote del amancay esta esencialmente ligado a la humedad relativa y el contenido de humedad del suelo, se refieren al momento en que los bulbos ya han completado su desarrollo interno.

Evaluación de la velocidad de rebrote según el tamaño de bulbo (grande, mediano y pequeño) y el momento de riego

Luego de iniciados los tratamientos de riego, el rebrote de los tres diferentes tamaños de bulbo se inició a las 38 semanas ( 9 meses) y finalizó luego de 7 semanas (10.5 riegos más tardíos fueron los que rebrotaron en menor tiempo. En bulbos donde se inició el riego luego de tres meses de plantados $\left(\mathrm{T}_{1}\right)$, se obtuvo el rebrote en un promedio de 277.5 días. Este tiempo fue significativamente menor que en bulbos regados luego de un mes $\left(\mathrm{T}_{3}\right)$, pero sin diferencias con los bulbos regados 2 meses después de plantados $\left(\mathrm{T}_{2}\right)$. Entre 1 mes $\left(\mathrm{T}_{3}\right)$ y 2 meses $\left(\mathrm{T}_{2}\right)$ tampoco hubo diferencias significativas. $A$ pesar que las diferencias no fueron significativas entre $T_{1}$ $\mathrm{y} \mathrm{T}_{2}$, y entre $\mathrm{T}_{2} \mathrm{y} \mathrm{T}_{3}$, los resultados revelan una progresión descendente en el número de días para el rebrote, conforme el momento de riego se fue retrasando. Estos resultados muestran que un mayor periodo de descanso permite completar el proceso de formación y desarrollo de los órganos foliares y florales al interior del bulbo en un menor tiempo. El retraso en el rebrote de los bulbos regados un mes después de plantados, pudo deberse a una reducción en la temperatura del medio de crecimiento por efecto del agua, lo que pudo generar una demora en el proceso de desarrollo interno del bulbo, o que no había transcurrido suficiente tiempo para completar los cambios internos. Sin embargo, el tratamiento donde los bulbos fueron regados luego de 3 meses de plantados $\left(\mathrm{T}_{1}\right)$, tuvo el mayor porcentaje de rebrote en menos tiempo.

\section{Bulbos medianos}

En bulbos medianos, se obtuvo los mismos resultados que en bulbos grandes. Un rebrote significativamente más rápido (275.3 días) en bulbos regados tres meses después de plantados $\left(\mathrm{T}_{1}\right)$, pero sin diferencias significativas con bulbos regados luego de 2 meses (284.7 días), pero con diferencias significativas con los de 1 mes $\left(\mathrm{T}_{3}\right)$. Entre los tratamientos regados 1 mes $\left(\mathrm{T}_{3}\right)$ y 2 meses $\left(\mathrm{T}_{2}\right)$ después de plantados, tampoco hubo diferencias significativas Nuevamente se observó que el rebrote de los bulbos en todos los tratamientos fue irregular, pero que el $\mathrm{T}_{1}$ predominó en porcentaje de bulbos rebrotados en menos días.

Tabla 5. Promedio de días para rebrotar en bulbos grandes en tres momentos de riego

\begin{tabular}{|c|c|c|c|c|c|c|c|c|}
\hline \multirow{2}{*}{$\begin{array}{l}\text { Tratamientos } \\
\text { de riego }\end{array}$} & \multicolumn{6}{|c|}{ Número de bulbos rebrotados } & \multirow{2}{*}{$\begin{array}{l}\text { Total bulbos } \\
\text { rebrotados }\end{array}$} & \multirow{2}{*}{$\begin{array}{l}\text { Promedio de días } \\
\text { para rebrotar }\end{array}$} \\
\hline & 269 días & 276 días & 283 días & 290 días & 304 días & 318 días & & \\
\hline $\mathrm{T}_{1}: 3$ meses & 13 & 0 & 3 & 1 & 0 & 2 & 19 & $277.5 \mathrm{a}$ \\
\hline $\mathrm{T}_{2}: 2$ meses & 3 & 6 & 1 & 4 & 1 & 5 & 20 & $290.0 \mathrm{ab}$ \\
\hline $\mathrm{T}_{3}: 1 \mathrm{mes}$ & 2 & 0 & 0 & 3 & 7 & 8 & 20 & $304.0 \mathrm{~b}$ \\
\hline
\end{tabular}

meses), con el rebrote del $100 \%$ de los bulbos grandes, del $83 \%$ de los bulbos medianos y del $78 \%$ de los bulbos pequeños.

\section{Bulbos grandes}

En la Tabla 5, se observa que los bulbos que recibieron
Otro aspecto a destacar, es que la velocidad de rebrote en bulbos medianos fue más rápida que en bulbos grandes para los tres tratamientos de riego. En el tratamiento de bulbos regados luego de 3 meses de plantados $\left(\mathrm{T}_{1}\right)$, el tiempo promedio de rebrote fue de 277.5 días en bulbos grandes y 275.3 días para bulbos medianos. Estos 
Tabla 6. Promedio de días del rebrote en bulbos medianos en tres momentos de riego

\begin{tabular}{|c|c|c|c|c|c|c|c|c|}
\hline \multirow{2}{*}{$\begin{array}{l}\text { Tratamientos } \\
\text { de Riego }\end{array}$} & \multicolumn{6}{|c|}{ Número de bulbos rebrotados en determinado número de días } & \multirow{2}{*}{$\begin{array}{l}\text { Total bulbos } \\
\text { rebrotados }\end{array}$} & \multirow{2}{*}{$\begin{array}{l}\text { Promedio de días } \\
\text { pare el rebrote }\end{array}$} \\
\hline & 269 días & 276 días & 283 días & 290 días & 304 días & 318 días & & \\
\hline $\mathrm{T}_{1}: 3$ meses & 11 & 4 & 1 & 2 & 1 & 0 & 19 & $275.3 \mathrm{a}$ \\
\hline $\mathrm{T}_{2}: 2$ meses & 6 & 2 & 5 & 0 & 1 & 3 & 17 & $284.7 \mathrm{ab}$ \\
\hline $\mathrm{T}_{3}: 1 \mathrm{mes}$ & 3 & 1 & 3 & 0 & 4 & 3 & 14 & $293.0 \mathrm{~b}$ \\
\hline
\end{tabular}

resultados coinciden con lo estudiado por Hanks y Jones (1987) en Hymenocallis x festalis, donde se indica que el crecimiento demoró más en los bulbos más grandes que en los más pequeños (43 días en los tamaños más pequeños y 49 días en los más grandes). Sin embargo, las razones por las cuales esto ocurre no han sido determinadas.

Como se observa, no todos los bulbos rebrotaron. Sin embargo, es importante destacar es que en el $\mathrm{T}_{1}$ solo el 5\% de los bulbos no rebrotó, mientras que en los tratamientos $\mathrm{T}_{2}$ y $\mathrm{T}_{3}$ el porcentaje fue mayor: $15 \% \mathrm{y}$ $30 \%$ respectivamente. Esta menor respuesta es debida al menor tamaño de los bulbos y a su menor cantidad de reservas.

Bulbos pequeños

En la Tabla 7, se observa que los tratamientos que tuvieron riegos más tardíos fueron los que más rápido rebrotaron. Sin embargo, no hubo diferencias significativas entre los bulbos regados a los tres $\left(\mathrm{T}_{1}\right)$, dos $\left(\mathrm{T}_{2}\right)$ y un mes de siembra $\left(\mathrm{T}_{3}\right)$. La tendencia en el promedio de días para el rebrote, fue similar a lo observado en bulbos grandes y medianos. Los bulbos regados luego de tres meses de el efecto sobre su floración. El mayor porcentaje de bulbos en flor se obtuvo con los de mayor tamaño, pero solo alcanzó el 12\%. En el caso de los bulbos medianos se obtuvo tan solo un 3\% y en bulbos pequeños no hubo floración.

En la Tabla 8, se observa que los bulbos grandes tuvieron un número significativamente superior de flores que en bulbos pequeños. No hubo diferencias con los bulbos medianos. Los resultados confirman lo señalado por Hartmann et al. (2002) que el tamaño y calidad de la flor está relacionado directamente con el tamaño del bulbo. El bulbo debe alcanzar un cierto tamaño mínimo para ser capaz de iniciar su primordio floral. De Hertogh y Zimmer (1989), mencionan que todas las plantas bulbosas deben alcanzar un tamaño mínimo de bulbo antes que puedan florear. Halevy (1990), indica también que el tamaño de bulbo generalmente determina si va producir flores o no. Los bulbos por debajo de un tamaño crítico, aun bajo condiciones inductivas, normalmente no logran florear. Hanks y Jones (1987) en su estudio sobre Hymenocallys $\mathrm{x}$ festalis, mencionan que solo un $25 \%$ de los bulbos

Tabla 7. Promedio de días para rebrotar en bulbos pequeños en tres momentos de riego

\begin{tabular}{ccccccccc}
\hline \multirow{2}{*}{$\begin{array}{c}\text { Tratamientos de } \\
\text { riego }\end{array}$} & 269 días & 276 días & 283 días & 290 días & 304 días & 318 días & Total & $\begin{array}{c}\text { Promedio de días } \\
\text { para rebrotar }\end{array}$ \\
\cline { 2 - 7 } $\mathrm{T}_{1}: 3$ meses & 10 & 3 & 0 & 2 & 1 & 3 & 19 & $281.9 \mathrm{a}$ \\
$\mathrm{T}_{2}: 2$ meses & 7 & 4 & 1 & 1 & 0 & 3 & 16 & $282.1 \mathrm{a}$ \\
$\mathrm{T}_{3}: 1$ mes & 4 & 1 & 1 & 0 & 2 & 4 & 12 & $292.9 \mathrm{a}$ \\
\hline
\end{tabular}

plantados $\left(\mathrm{T}_{1}\right)$ fueron los que rebrotaron más rápido, seguido por el tratamiento de dos meses $\left(\mathrm{T}_{2}\right)$ y finalmente por el tratamiento de un mes $\left(\mathrm{T}_{3}\right)$.

Nuevamente se sugiere, que los riegos más tempranos tuvieron un efecto sobre la temperatura del medio de crecimiento, lo que a su vez retrasó el crecimiento de primordios foliares y florales, lo que finalmente hizo que el rebrote fuera más tardío.

\section{Evaluación de la floración en "amancay" según el} tamaño de bulbo

Se comparó independientemente los tres tamaños de bulbo, sin considerar el momento de riego, para observar pequeños $(9-10 \mathrm{~cm})$ produjeron flores, pero en bulbos grandes $(>13 \mathrm{~cm})$ el 90\% como mínimo lo logró. Otra posible explicación con respecto al bajo porcentaje de floración en los bulbos de diferente tamaño puede ser que el experimento fue realizado bajo sombra $(50 \%$ de sombra). Theron y Jacobs (1996) mencionan que en Nerine bowdenii (Amaryllidaceae), el sombreamiento durante cualquier período de crecimiento redujo el porcentaje de floración en comparación con el testigo ( $\sin$ sombreamiento). El colocar plantas en sombra durante la primera etapa de crecimiento (4 meses), produjo sólo un $33 \%$ de los bulbos de floración en comparación con 
un 58\% que floreció cuando se colocó sombra sobre la segunda mitad de crecimiento.

Tabla 8. Número de bulbos en floración según su Tamaño

\begin{tabular}{cc}
\hline Tamaño de Bulbo & Numero de Bulbos con flor \\
\hline Grandes & $7 \mathrm{a}$ \\
Medianos & $2 \mathrm{ab}$ \\
Pequeños & $0 \mathrm{~b}$ \\
\hline
\end{tabular}

\section{Evaluación de la floración en "amancay" según el momento de riego}

Se comparó independientemente los tres momentos de riego, sin considerar el tamaño de bulbo, para determinar su efecto sobre la floración. Los bulbos que tuvieron mayor floración fueron aquellos que recibieron riegos luego de tres meses de reposo $\left(\mathrm{T}_{1}\right)$, seguido por los bulbos que recibieron riego de dos meses $\left(\mathrm{T}_{2}\right)$. Sin embargo, en ambos tratamientos, el porcentaje de bulbos con flor fue bajo $(35 \%$ y $10 \%)$. El tratamiento $\mathrm{T}_{3}$, no tuvo floración (Tabla 9).

Tabla 9. Número de bulbos en floración según el momento del riego

\begin{tabular}{cc}
\hline Momento de Riego & $\begin{array}{c}\text { Numero de Bulbos con } \\
\text { flor }\end{array}$ \\
\hline $\mathrm{T}_{1}: 3$ meses & $7 \mathrm{a}$ \\
$\mathrm{T}_{2}: 2$ meses & $2 \mathrm{ab}$ \\
$\mathrm{T}_{3}: 1$ mes & $0 \mathrm{~b}$ \\
\hline
\end{tabular}

Brito (2006), menciona que el proceso de floración involucra cinco pasos sucesivos: inducción, iniciación, organogénesis, maduración (crecimiento de las partes florales) y antesis. Estos pasos son controlados por varios factores y cada uno de ellos tiene lugar en un determinado periodo del ciclo de crecimiento. Este proceso de floración puede ser controlado por la aplicación de tratamientos específicos al bulbo (temperatura, humedad, luz y reguladores de crecimiento).

En el presente experimento se muestra que el factor humedad, a través de diferentes momentos de riego, tuvo influencia sobre la floración. Sin embargo, el mecanismo por el cual el momento de riego logra promover una mayor floración no tiene una explicación clara. Lo que sí parece evidente es que en alguna parte del proceso de floración, provocado por un riego demasiado prematuro, se presentó algún factor negativo que actuó sobre la inducción, iniciación u organogénesis, dando como resultado una menor floración.
Evaluación de la floración según el tamaño de bulbo y el momento de riego

La mayor floración se obtuvo en los bulbos de tamaño grande, pero solo en los que recibieron riego a los tres meses de siembra $\left(T_{1}\right)$. En bulbos medianos y pequeños la floración fue insignificante o no se presentó. En bulbos grandes, los que tuvieron mayor número de flores fueron aquellos que tuvieron un mayor tiempo de reposo ( 3 meses) antes de ser regados, pero solo tuvo diferencias significativas con los bulbos pequeños (Tabla 10).

Tabla 10. Número de bulbos grandes en floración según el momento después del riego

\begin{tabular}{|c|c|c|c|c|c|c|c|}
\hline \multirow{2}{*}{$\begin{array}{c}\text { Tratamientos } \\
\text { de riego }\end{array}$} & \multicolumn{6}{|c|}{$\begin{array}{l}\text { Bulbos en flor a un } \\
\text { determinado número de días }\end{array}$} & \multirow{2}{*}{$\begin{array}{l}\text { Total } \\
\text { bulbos } \\
\text { en flor }\end{array}$} \\
\hline & $\begin{array}{l}269 \\
\text { días }\end{array}$ & $\begin{array}{l}276 \\
\text { días }\end{array}$ & $\begin{array}{l}283 \\
\text { días }\end{array}$ & $\begin{array}{l}290 \\
\text { días }\end{array}$ & $\begin{array}{l}304 \\
\text { días }\end{array}$ & $\begin{array}{l}318 \\
\text { días }\end{array}$ & \\
\hline $\mathrm{T}_{1}: 3$ meses & 1 & 0 & 1 & 1 & 2 & 0 & $5 \mathrm{a}$ \\
\hline $\mathrm{T}_{2}: 2$ meses & 1 & 0 & 0 & 1 & 0 & 0 & $2 a b$ \\
\hline $\mathrm{T}_{3}: 1 \mathrm{mes}$ & 0 & 0 & 0 & 0 & 0 & 0 & $0 \mathrm{~b}$ \\
\hline
\end{tabular}

Tabla 11. Número de bulbos medianos en floración según el momento después del riego

\begin{tabular}{|c|c|c|c|c|c|c|c|}
\hline \multirow{2}{*}{$\begin{array}{l}\text { Tratamientos } \\
\text { de riego }\end{array}$} & \multicolumn{6}{|c|}{$\begin{array}{l}\text { Bulbos en flor a un } \\
\text { determinado número de días }\end{array}$} & \multirow{2}{*}{$\begin{array}{c}\text { Total } \\
\text { bulbos } \\
\text { en flor }\end{array}$} \\
\hline & $\begin{array}{l}269 \\
\text { días }\end{array}$ & $\begin{array}{l}276 \\
\text { días }\end{array}$ & $\begin{array}{l}283 \\
\text { días }\end{array}$ & $\begin{array}{l}290 \\
\text { días }\end{array}$ & $\begin{array}{l}304 \\
\text { días }\end{array}$ & $\begin{array}{l}318 \\
\text { días }\end{array}$ & \\
\hline $\mathrm{T}_{1}: 3$ meses & 0 & 1 & 0 & 0 & 1 & 0 & $2 \mathrm{a}$ \\
\hline $\mathrm{T}_{2}: 2$ meses & 0 & 0 & 0 & 0 & 0 & 0 & $0 \mathrm{a}$ \\
\hline $\mathrm{T}_{3}: 1 \mathrm{mes}$ & 0 & 0 & 0 & 0 & 0 & 0 & $0 \mathrm{a}$ \\
\hline
\end{tabular}

Tabla 12. Número de bulbos pequeños en floración según el momento después del riego

\begin{tabular}{|c|c|c|c|c|c|c|c|}
\hline \multirow{2}{*}{$\begin{array}{l}\text { Tratamientos } \\
\text { de riego }\end{array}$} & \multicolumn{6}{|c|}{$\begin{array}{l}\text { Número de bulbos en flor a un } \\
\text { determinado número de días }\end{array}$} & \multirow{2}{*}{$\begin{array}{c}\text { Total } \\
\text { bulbos } \\
\text { en flor }\end{array}$} \\
\hline & $\begin{array}{l}269 \\
\text { días }\end{array}$ & $\begin{array}{l}276 \\
\text { días }\end{array}$ & $\begin{array}{l}283 \\
\text { Días }\end{array}$ & $\begin{array}{l}290 \\
\text { días }\end{array}$ & $\begin{array}{l}304 \\
\text { días }\end{array}$ & $\begin{array}{l}318 \\
\text { días }\end{array}$ & \\
\hline $\mathrm{T}_{1}: 3$ meses & 0 & 0 & 0 & 0 & 0 & 0 & $0 \mathrm{a}$ \\
\hline $\mathrm{T}_{2}: 2$ meses & 0 & 0 & 0 & 0 & 0 & 0 & $0 \mathrm{a}$ \\
\hline $\mathrm{T}_{3}: 1 \mathrm{mes}$ & 0 & 0 & 0 & 0 & 0 & 0 & $0 \mathrm{~b}$ \\
\hline
\end{tabular}

En los bulbos medianos y pequeños, la floración no fue significativa en ninguno de los momentos de riego (Tablas 11 y 12).

Nuevamente se observa que, en el caso de bulbos grandes, el factor momento de riego tuvo influencia sobre la iniciación floral. En el caso de los bulbos medianos 
y pequeños, el factor más importante, fue el tamaño del bulbo y su cantidad de reservas, ya que los medianos florearon muy poco y los pequeños no lo hicieron.

\section{Conclusiones}

Tres tamaños de bulbo de amancay (Ismene amancaes), regados tres y dos meses después de plantados, lograron rebrotar en un tiempo significativamente menor, que aquellos regados un mes después. Los bulbos que fueron regados luego de tres meses de plantados, fueron los que más florearon, pero solo tuvieron diferencias significativas con los regados luego de un mes. Con respecto al tamaño de bulbo, los más grandes tuvieron un porcentaje mayor de floración, mostrando diferencias significativas con los bulbos pequeños, más no con los medianos. En ninguno de los tratamientos, el tiempo de rebrote luego del riego fue menor a los 270 días (9 meses), periodo necesario para la diferenciación y desarrollo de estructuras vegetativas y reproductivas al interior del bulbo. Una vez finalizado este periodo, el manejo del tiempo de riego fue un factor determinante para activar el rebrote de los bulbos de amancay.

\section{Literatura citada}

Aguilar, P. 1974. Apreciaciones sobre la vegetación y flora de las lomas del Morro Solar, Tablada de Lurín y Atocongo. Anales Cientificos, 12 (1-2), 79-84.

Agüero, S. 2002. Influencia de humedad edáfica en el ciclo biológico y propagación de Ismene amancaes $R \& P$. Herbert "amancaes" (Amaryllidaceae) en condiciones "in situ" y "ex situ". (Tesis para optar el título profesional de Biólogo). Universidad Nacional Mayor de San Marcos. Lima. Perú.

Agüero, S. y Suni, M. 1999. Influencia del evento "El Niño 1997 - 1998" en el desarrollo de Ismene amancaes (Amaryllidaceae, Liliopsidae). En: El Niño 1997 - 98 y su impacto sobre los ecosistemas marino y terrestre. Revista Peruana de Biología. Vol. Extraordinario,118 124.

Brack, A. 1999. Diccionario enciclopédico de plantas útiles del Perú. CBC - Centro de Estudios Regionales Andinos "Bartolomé de las Casas". Cuzco.

Borys, M., Leszcynska-Borys, H., and Galvan, J. 2008. Variants in Hymenocallys Salisb. Acta Horticulturae, 766, 391-398.

Borys, M., Leszcynska-Borys, H., and Galvan, J. 2009. Hymenocallis Salisb. - Bulbs reaction to applied temperatures. Acta Horticulturae, 813, 631-636.

Brito, C. 2006. Morphological analysis of tropical bulbs and environmental effects on flowering and bulb development of Habranthus and Zephyranthes spp. (Tesis de Maestría). Universidad de Florida.

Cano, A., Roque, J., Arakaki, M., Arana, C., La Torre, M., Llerena, N. y Refulio. N. 1999. Diversidad florística de las Lomas de Lachay (Lima) durante el evento "El Niño 1997 - 1998”. Revista Peruana de Biología. Vol. Extr., 125-132.

Carrillo, R. 2004. Apuntes de la flor de amancaes. The
Biologist. 2 (2:6).

Dafni, A., Cohen, D., and Noy-Meir, I. 1981. Lifecycle variation in geophytes. Annual Missouri Botanical Garden, 68, 652-660.

De Hertogh, A., and Le Nard, M. 1993. The physiology of flower bulbs. Elsevier Science. Hillegom, Holanda.

De Hertogh, A. 1996. Holland bulb forcer's guide. 5ta. Edición. Intl. Flower Bulb Centre, Hillegom, Holanda.

De Hertogh, A., and Zimmer, K. 1989. Allium Ornamental Species. En: Handbook of Flowering, Vol. VI. Ed.: A. Halevy. CRC Press. Pp 22 - 33.

Ferreyra, R. 1979. Sinopsis de la flora peruana. Boletín de Lima, 1 (1), 1-59.

Halevy, A. 1990. Recent advances in control of flowering and growth habit of geophytes. Acta Horticulturae. 266, $35-42$.

Hanks, G., and Jones, S. 1987. The growth and flowering of Hymenocallis x festalis. Scientia Horticulturae, 30, 301-313.

Hartmann, H., Kester, D., Davies, F., and Geneve, R. 2002. Plant Propagation: Principles and Practices. 7a Ed. Prentice Hall, N.J.

Kamenetsky, R. 1994. Life cycle, flower initiation and propagation of the desert geophyte Allium rothii. International Journal of Plant Sciences, 155(5), 597-605. Kamenetsky, R., and Okubo, H. 2013. Ornamental Geophytes: From Basic Science to Sustainable Production. CRC Press.

Le Nard, M. and De Hertogh, A. 2002. Research needs for flower bulbs (geophytes). Acta Horticulturae, 570, 121-127.

Morales, P., Schiappacasse, F., Peñalillo, P., and Yañez, P. 2009. Effect of bulb weight on the growth and flowering of Herbertia lahue subsp. lahue (Iridaceae). Ciencia e Investigación Agraria, 36(2),259-266.

Mori, G., Imanishi, H., and Sakanishi, Y. 1991. Effect of temperature on flowering of Hymenocallis speciosa Salisb. J. Japan Soc. Hort. Sci. 60(2), 387-393.

Ochoa, R. 2008. La fiesta inolvidable: Flor de Amancaes en Huarochirí. Revista Rumbos de Sol \& Piedra, 52,4860.

Rossa, B., and Von Willert. D. 1999. Physiological characteristics of geophytes in semi-arid Namaqualand South Africa. Plant Ecology, 142, 121-132.

Rees, A. R. 1972. The growth of bulbs. Academic Press. London \& New York. 291 pp.

Ress, A. R. 1992. Ornamental bulbs, corms and tubers. CAB Intenational. UK. 220 pp.

Suni, M., Pascual, E. y Jara, E. 2011. Desarrollo reproductivo del "amancay" Ismene amancaes (Amaryllidaceae) en su ambiente natural. Revista Peruana de Biología, 18(3),293-297.

Theron, K., and De Hertogh, A. 2001. Amaryllidaceae: Geophytic growth, development and flowering. Horticultural Reviews, 25, 1-70.

Theron, K., and Jacobs, G. 1996. The Effect of irradiance, defoliation, and bulb size on flowering of Nerine bowdenii W. Watson (Amaryllidaceae). J. Amer. 
Soc. Hort. Sci. 121(1),115-122

Torres, J. y López, C. 1981. Productividad primaria en las Lomas de la Costa Central del Perú. Boletín de Lima, 14,1-11.

Webwebauer, A. 1945. El mundo vegetal de los andes peruanos, Estudio Fitogeográfico. Lima: Ministerio de Agricultura.

Wang, Y. T., and Roberts, A. N. 1983. Influence of air and soil temperatures of Lillium longiflorum Thunb. During different growth phases. J. Amer. Soc. Hort. Sci. $108,810-815$.

Wiebe, H. J. 1994. Effects of temperature and daylength on bolting of leek (Allium porrum L.). Sci. Hort., 59, 177-185. 